\title{
Pulsed Depositions vs. Continuous Growth: Monte Carlo Study of Submonolayer Regime*
}

\author{
Martin Mašín and Miroslav Kotrla ${ }^{\dagger}$ \\ Institute of Physics, Academy of Sciences of the Czech Republic, \\ $\mathrm{Na}$ Slovance 2, 182 21, Praha 8, Czech Republic
}

(Received 9 September 2009; Accepted 20 January 2010; Published 13 February 2010)

\begin{abstract}
We present theoretical study comparing kinetics, and the time evolution of surface features for two modes of epitaxial growth - pulsed deposition with a given repetition rate (PLD mode), and continuous growth with a constant flux (MBE mode). We confine ourselves to the sub-monolayer regime. We study island morphology and analyze island density as a function of the substrate temperature and the coverage. We show that the behavior of the island density as a function of the temperature in both cases is quite different. The island densities in the PLD mode are substantially higher than in the MBE mode, provided the temperature is sufficiently high. In the case of PLD, we observe anomalous temperature dependence of the island density in a certain temperature interval. We analyzed the temperature dependence of concentration of small island, and we found that anomalous behavior is related to a persistence of small surface clusters during PLD growth. Anomaly is not present in MBE growth, because the concentration of small islands in this mode of growth is very low. The problem is studied by kinetic Monte Carlo simulations employing the full diffusion solid-on-solid model with specific material parameters based on a recent experiment. [DOI: 10.1380/ejssnt.2010.65]
\end{abstract}

Keywords: Models of non-equilibrium phenomena; Epitaxy; Pulsed laser deposition; Surface diffusion; Clusters; Monte Carlo simulations; Solid-gas interfaces

\section{INTRODUCTION}

Pulsed laser deposition (PLD) has been extensively utilized in the past for the preparation of multicomponent oxide films [1]. It was in particular used for epitaxial growth of dielectric oxides on the direct gap semiconductors, an example of such material is Y-stabilized $\mathrm{ZnO}_{2}$ on InP (YSZ/InP) [2] (see references for other materials of this type therein). But, it is recently being employed also for the preparation of magnetic thin films, e.g. $\mathrm{Fe} / \mathrm{Cu}(111)$ [3], or $\mathrm{Fe}$ on $\mathrm{W}$, or on Mo $[4,5]$. Hence, PLD is becoming the interesting alternative technique to molecular beam epitaxy (MBE) that has been widely used for metal as well as semiconductor epitaxy. However, from the theoretical point of view PLD growth is significantly less understood than MBE growth which has been intensively studied in the past. Whereas a ratio $D / F$ between diffusion coefficient $D$ and deposition flux $F$ is the main parameter controlling MBE growth, PLD is characterized by more parameters. In addition to the ratio $D / F$, also by chopping frequency $f$ of pulsed deposition (frequency of switching of the flux on and off), and by fractions of a period between pulses during which particles are deposited or allowed to relax.

In this paper, we compare kinetics and morphology of surfaces features for two above mentioned modes of epitaxial growth in the case of a submonolayer system. This study is motivated by the recent PLD experiment [6]. We do not attempt to describe complex processes in beam of particles before and during PLD, but we concentrate on effect of microscopic processes at the surface on kinetics and morphology of surface growth. We demonstrate

\footnotetext{
*This paper was presented at 10th International Conference on Atomically Controlled Surfaces, Interfaces and Nanostructures (ACSIN-10), Granada Conference Centre, Spain, 21-25 September, 2009 .

†Corresponding author: kotrla@fzu.cz
}

important role of stability of small compact clusters for growth at different temperatures. System is investigated by lattice Monte Carlo simulation. We employ the full diffusion model which takes into account processes of nucleation, island growth, as well as island decay.

\section{MODEL}

In our simulations, we use the solid-on-solid model of epitaxial growth [7] that has been shown to be capable to reproduce many aspects of epitaxial growth. The substrate is assumed to have simple cubic structure, neither vacancies nor overhangs are permitted. Incoming particles are deposited at random positions, desorption is forbidden. The direct application of the SOS model for heteroepitaxial system with lattice mismatch is problematic. In the case of a multilayer lattice system with mismatch, one would need to introduce additional variables describing surface deformation. However, we restrict here to the submonolayer growth on singular surface with low coverage. We believe that in this case the SOS model can provide good approximative description.

PLD growth is modeled by modulation of the incident flux by alternation between two states: i) the fast deposition during individual pulses, flux has nonzero average value $F_{\mathrm{A} V}$ in the time interval $\Delta t_{\mathrm{dep}}$, and ii) no deposition, only the relaxation of the system between pulses for the time interval $\Delta t_{\text {diff. }}$. During the relaxation, particles only diffuse on the surface. Therefore, we call this interval a diffusion period. Note that the diffusion proceeds also during the deposition, but because $\Delta t_{\text {dep }} \ll \Delta t_{\text {diff }}$ this contribution is very small. Pulses are repeated with the chopping frequency $f=1 /\left(\Delta t_{\text {dep }}+\Delta t_{\text {diff }}\right)$. We do not include any spatial correlation of particles in a pulse. In the PLD simulations presented here, we have use time and deposition parameters corresponding to the PLD experiment for $\mathrm{Fe} /$ Mo system $[6], \Delta t_{\text {dep }}=10 \mu \mathrm{s}$, and $f=10$ $\mathrm{Hz}$. In the case of MBE, flux of deposited particles $F$ is continuous, we used $F=0.005 \mathrm{ML} / \mathrm{s}$ in presented simu- 


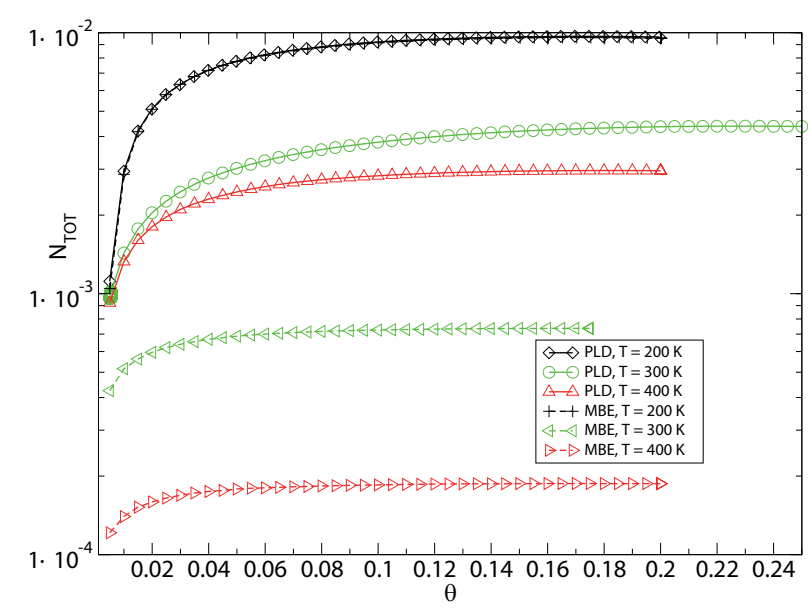

FIG. 1: Total island density as function of the coverage for three different temperatures $200 \mathrm{~K}, 300 \mathrm{~K}, 400 \mathrm{~K}$ and both growth modes MBE and PLD. Simulations were stopped when the system reached the saturation coverage $\theta_{\text {sat }}$.

lations. The same value of an average flux $F_{\mathrm{A} V}$ is used in PLD mode.

The diffusion is modeled by thermally activated jumps of adatoms to the nearest neighbor position with the configuration dependent Arrhenius-type rate. The probability of a jump of an adatom from site $i$ is exponentially proportional to the product of activation energy $E_{\mathrm{A}(i)}$ and the inverse temperature $1 / T$

$$
P_{i} \sim \exp \left(-\frac{E_{\mathrm{A}(i)}}{k_{B} T}\right),
$$

here $k_{B}$ is the Boltzmann constant. Total activation energy $E_{\mathrm{A}(i)}$ for chosen adatoms is given by sum of activation energy for free particle $E_{d}$ and additive interaction of an adatom with particles present in the nearest neighbor positions of the site $i$

$$
E_{A}(i)=E_{d}+l E_{b}
$$

where $l \in\langle 0,4\rangle$ is the number of occupied nearest neighbor sites of the site $i$, and $E_{b}$ is the binding energy. The rates are formally normalized to unity, however, in practical implementation of KMC simulation only relative rates are utilized. In general, the temperature and interaction energies are regarded as variables. But for all simulations in this paper, we have set $E_{d}=0.4 \mathrm{eV}$ - typical value for self-diffusion on metal surfaces, to mimic interaction of $\mathrm{Fe}$ on $\mathrm{Mo}(110)$, we set $E_{b}=0.6 \mathrm{eV}$. In our KMC simulation we utilized the BKL algorithm with the implementation by Maksym [8]. Reported results represent averages over at least 10 simulations on a lattice of size ranging from $500 \times 500$ to $1000 \times 1000$ sites.

\section{RESULTS AND DISCUSSION}

Submonolayer island density $N_{\text {TOT }}(\theta)$ in the beginning of the deposition increases as a power. With the continuing deposition one observes a plateau or a maximum of island density. $N_{\text {TOT }}(\theta)$ saturates to its stationary value, and finally it decreases due to coalescence of islands. A coverage corresponding to the beginning of the plateau

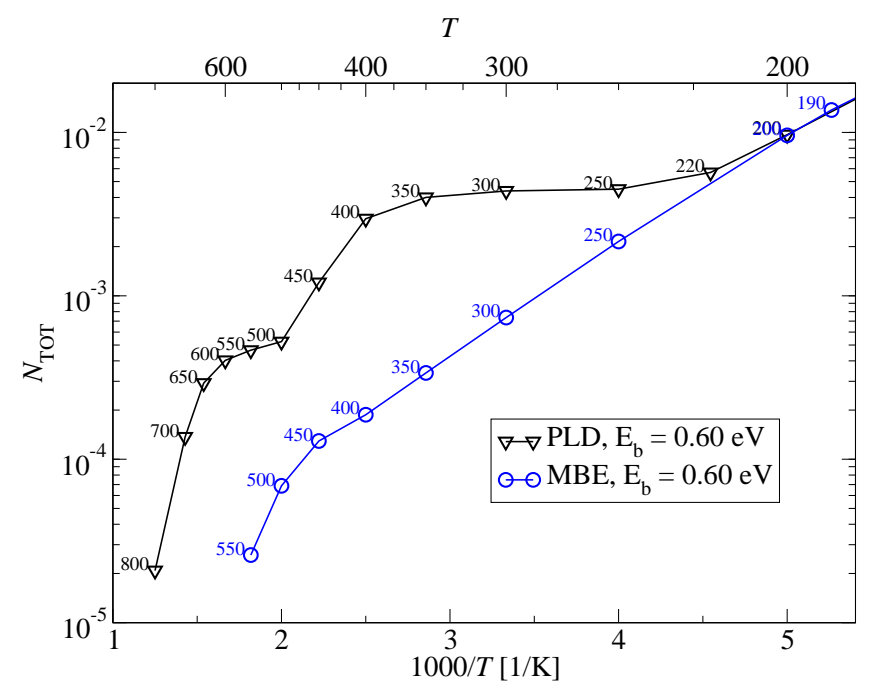

FIG. 2: Comparison of MBE and PLD island densities as function of the temperature.

(or the maximum) of the submonolayer island density is called the saturated coverage $\theta_{\text {sat }}$. The island density is measured in experiment or in simulations usually in a stationary state, where it has the plateau. Therefore, one needs to assess the saturation coverage. Figure 1 displays the dependence of $N_{\mathrm{TOT}}(\theta)$ on the coverage $\theta$ for both modes, and for three different temperatures. There is no difference between the PLD and MBE growth for the low temperature (below $200 \mathrm{~K}$ ). However, for the higher temperatures the island density for the PLD mode is much larger than for the MBE mode. The saturation coverage is reached in a similar way for both modes. One can see some difference for the temperature $T=300 \mathrm{~K}$, it will be commented below. The value of the saturated coverage is rapidly decreasing with the increasing temperature. The typical value of $\theta_{\text {sat }}$ in the case of PLD is approximately $0.2 \mathrm{ML}$, at $T=200 \mathrm{~K}$ and only $0.01 \mathrm{ML}$ at $T=750 \mathrm{~K}$.

In Fig. 2, we show the total island density $N_{\text {TOT }}$ as a function of the inverse temperature (Arrhenius plot) again for both modes. We observe that in the case of MBE there is, with the very good approximation, the linear behavior with the inverse temperature in the interval from $200 \mathrm{~K}$ to $400 \mathrm{~K}$, and that for higher temperatures there is a monotonous change of slope of the curve related to a change of the critical island size. In the case of PLD, the situation is more complex. We observe that for $\mathrm{T}<$ $200 \mathrm{~K}$ the island density in the PLD mode is the same as in the MBE mode, and we can also see that the PLD curve is approximately linearly dependent on the inverse temperature at low and high temperatures: $200 \mathrm{~K}-400 \mathrm{~K}$ and above $650 \mathrm{~K}$. However, there are some differences. First of all, we can see that for all temperatures above $200 \mathrm{~K}$ values for the PLD mode are higher than values for the MBE mode. Similar situation has been already noted in a simulation of the simplified irreversible model [9]. But in addition, we observe the anomalous temperature dependence with a non-monotonous change of the slope in some interval of intermediate temperatures, from $500 \mathrm{~K}$ to $650 \mathrm{~K}$.

In order to understand the situation microscopically, we investigated also similarities and differences in island morphologies for both growth regimes. The overall pic- 

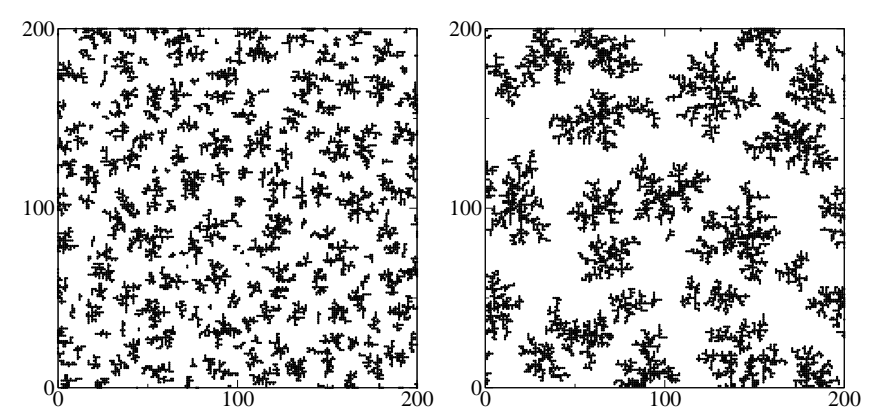

FIG. 3: Examples of surface configurations in the regime of the saturation for PLD (left) and MBE (right) at $300 \mathrm{~K}$.
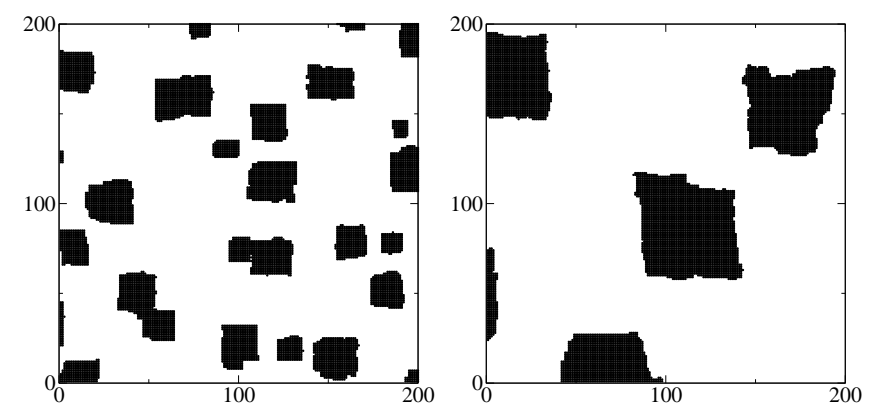

FIG. 4: Examples of surface configurations in the regime of the saturation for PLD (left) and MBE (right) at $500 \mathrm{~K}$.

ture is obtained when one looks on configurations in the regime of saturation. Whereas for the temperature $\mathrm{T} \leq$ $200 \mathrm{~K}$ configurations for both modes contain very similar fractal features, with the increasing temperature we can see a difference. It should be mentioned that the curve for PLD splits from the curve for MBE just at $200 \mathrm{~K}$. Figures 3 and 4 illustrate surface configurations in the regime of the saturation for MBE and PLD growth at higher temperatures $300 \mathrm{~K}$ and $500 \mathrm{~K}$ respectively. Fractals are still observed for the both modes at the temperature $300 \mathrm{~K}$, but for the MBE mode fractals have larger lateral size. In the case of PLD, new islands are being nucleated also for relatively high coverage. That is why saturation coverage is increased in PLD mode at $T=300 \mathrm{~K}$ (cf. Fig. 1). In general, shape of islands starts to change to more and more compact with the increasing temperature. Fractal features are reduced for both modes due to the increasing diffusion of atoms along edges with the increasing temperature. Hence, we observe the compact square islands for higher temperature. The difference in the level of island density is also clearly demonstrated in these static pictures.

One can learn more if one follows the time evolution which is illustrated in Fig. 5. During the deposition new islands are nucleated in both modes, however, process of nucleation is enhanced in the PLD mode. The detailed inspection shows that in the case of PLD, islands of sizes 2, $6,8,9$ are often created after a pulse at certain temperatures. These islands serve as immobile nucleation centers. We analyze effect of these clusters at different temperatures below.

It is known that MBE growth is substantially controlled by the concentration of monomers. In order to better understand the difference between MBE and PLD growth, we studied the temperature dependence of the concen-

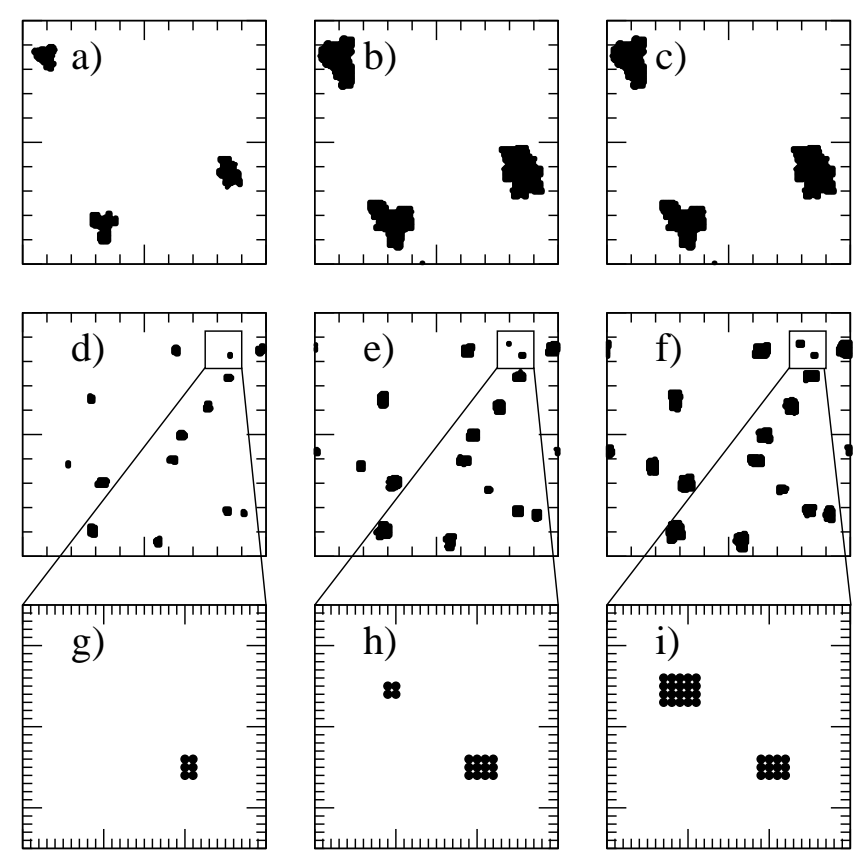

FIG. 5: Examples of development of surface configurations. Figs. a)-c) represent the development of the MBE system at the coverage $\theta=0.010,0.025$ and 0.040 . The development of PLD system at same coverage as in Figs. a)-c) is plotted in Figs. d)-f). The size of presented sections is $200 \times 200$ sites. In the case of PLD, detailed views of selected small clusters in particle resolution are displayed in Figs. g)-i).

tration of monomers and small clusters for both modes. Figure 6 displays density of monomers and small clusters of selected sizes with 2, 4, and 7 atoms as a function of the temperature for PLD as well as for MBE. This scan is obtained for the early stage of the deposition, after $1 \mathrm{~s}$. In the case of MBE, we can see that monomers as well as small island practically decay at $T=300 \mathrm{~K}$. Curves for these islands above $300 \mathrm{~K}$ collapse into one curve (see left-down corner in the inset). We continuously deposit ever new monomers in the MBE mode but mobility of monomer at this temperature is so high that practically all monomers are attached to existing islands, probability of nucleation of a new island is very low.

We observe different situation in the case of PLD. First of all, the concentration of monomers for the PLD mode is also rapidly decaying to very low values at $T \geq 250 \mathrm{~K}$. The value at $T=250 \mathrm{~K}$ is even lower than the monomer concentration for the MBE mode. This decrease is more apparent in the temperature interval 250-300 K. This effect is caused by the facts that, (i) we scan PLD system at the end of the diffusion period and (ii) the mobility of monomers at the given temperature is so high that almost all monomers can attach to existing islands or create dimers during the diffusion period.

More significant difference of the PLD mode is that the concentration of dimers and all larger clusters in the case of PLD is remarkably higher. Mainly high stability and low diffusivity of dimers in the comparison with high diffusivity of monomers is responsible for high increased saturation coverage at temperatures around $300 \mathrm{~K}$. The concentrations of small islands depend only weakly on the temperature in the interval $T=250-450 \mathrm{~K}$, in this early stage of the deposition. Notice also that in this in- 


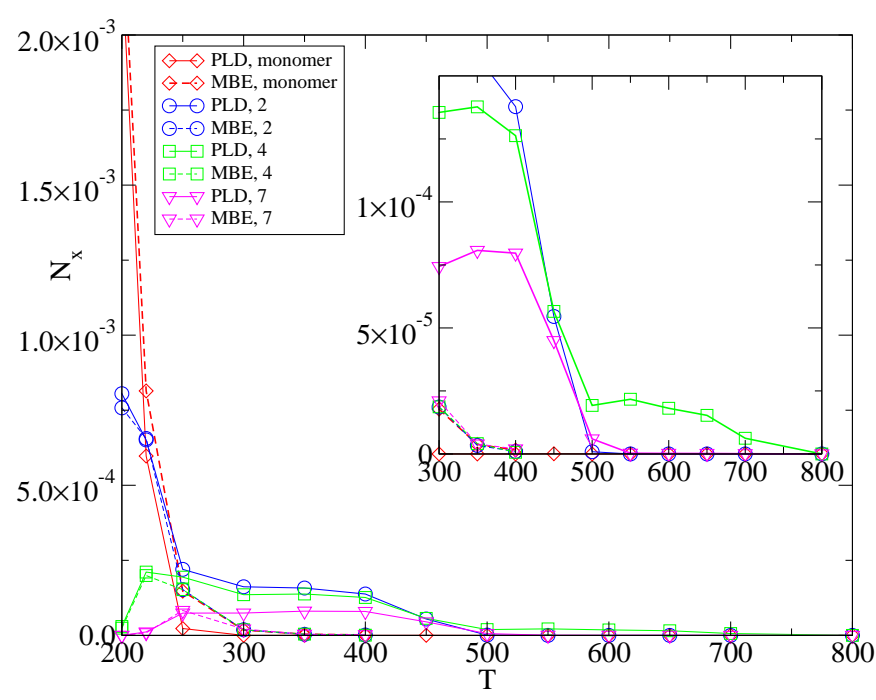

FIG. 6: Concentrations of monomers and small clusters of selected sizes with 2, 4, and 7 atoms after $1 \mathrm{~s}$ of the deposition (10 pulses in the case of PLD) as function of the temperature. In the case of PLD, concentrations are shown at the end of the diffusion period, i.e. at a moment when a new pulse is approaching the surface. Inset: Detail for intermediate and high temperatures.

terval, the concentrations of small islands is reciprocally proportional to the island size. Above the temperature $T=400 \mathrm{~K}$, dimers decay. Hence, based on the comparison with the known behavior of MBE growth, we can conclude that dimers play here a role of critical island size up to the temperature $T \approx 400 \mathrm{~K}$ and they are responsible for a plateau between $250 \mathrm{~K}$ and $400 \mathrm{~K}$ in the temperature dependence of the island density for PLD. At temperatures $T>500 \mathrm{~K}$ the critical island size increase to $i=4$, and we observe a new plateau in the dependence of the island density for PLD at temperature interval $500 \mathrm{~K}<T<650 \mathrm{~K}$. We observe persistent stability of island of size 4 at this temperature interval (see Fig. 6), whereas other islands, like e.g. islands of size 7 almost decay. Origin of this selected stability is subject of our ongoing research.

\section{CONCLUSION}

We have presented the results of simulation of two modes of epitaxial growth: pulsed laser deposition with the chopped flux, and continuous growth with the constant flux as in molecular beam epitaxy. We investigated the island morphology and the dependence of island density on the temperature and the coverage for both modes in the submonolayer regime. The values of saturated island density rapidly decrease with the increasing temperature. The comparison of the time evolution of surface features reveals the increased nucleation and the persistence of small islands in PLD mode. Therefore, the island density in PLD mode is substantially higher than in MBE mode, provided the temperature is sufficiently high.

The analysis of the temperature dependence of saturated island densities further confirms the substantial difference of both modes. In the case of PLD, we observe the anomalous temperature dependence of the island density in a certain temperature interval. We anticipate that this phenomenon is related to generation of an excess of small stable clusters. It would be interesting to calculate island size distribution function in more details and explore existence of scaling. Further work on this phenomena is in the progress.

\section{Acknowledgments}

This work was done within the project AV0Z1-010 0520 of the ASCR. Authors acknowledge the support from the Grant Agency of the Czech Republic under Contract No. 202/09/0775, and by joint funding under EU STRP 016447 MagDot and NSF DMR Award No. 0502737.
[1] D. B. Chrisey and G. K. H. (ed), Pulsed Laser Deposition of Thin Films (Wiley, New York, 1994).

[2] E. Vasco and C. Zaldo, J. Phys.: Condens. Matter 16, 8201 (2004).

[3] H. Jenniches, M. Klaua, H. Höche, and J. Kirschner, Appl. Phys. Lett. 69, 3339 (1996).

[4] P.-O. Jubert, O. Fruchart, and C. Mayer, Phys. Rev. B 64, 115419 (2001).

[5] O. Fruchart, P.-O. Jubert, M. Eleoui, F. Cheynis, B. Borca, P. David, V. Santonacci, A. Lienard, M. Hasegawa, and
C. Mayer, J. Phys.: Condens. Matter 19, 53001 (2007).

[6] P.-O. Jubert, O. Fruchart, and C. Mayer, Surf. Sci. 522, 8 (2003).

[7] S. Clarke and D. D. Vvedensky, J. Appl. Phys. 63, 2272 (1988) .

[8] P. A. Maksym, Semicond. Sci. Technol. 3, 594 (1988).

[9] B. Hinnemann, H. Hinrichsen, and D. Wolf, Phys. Rev. Lett. 87, 135701 (2001). 